\title{
Valeur alimentaire du lupin blanc (Lupinus albus var Lutop) chez la truite arc-en-ciel (Oncorhynchus mykiss). Effet de la cuisson-extrusion
}

\author{
D Bangoula, JP Parent, F Vellas* \\ Laboratoire d'Écophysiologie des poissons, université Paul-Sabatier, 38, rue des 36-Ponts, \\ 31078 Toulouse cedex, France
}

(Reçu le 10 septembre 1992; accepté le 28 mai 1993)

\begin{abstract}
Résumé - Deux expériences furent réalisées pour déterminer la qualité nutritionnelle, pour la truite, d'aliments renfermant, comme source partielle de protéines, de la farine de lupin "Lutop" crue (LC) ou extrudée à $120^{\circ} \mathrm{C}$ (LE1) et à $145^{\circ} \mathrm{C}$ (LE2). La première fut consacrée à la mesure du coefficient d'utilisation digestive apparent (CUDa) de régimes avec 20 et $30 \%$ de lupin ; la seconde, à l'étude de l'influence d'un aliment contenant $20 \%$ de lupin sur les performances zootechniques. Les résultats démontrent que :

- le CUDa de la matière sèche, des protéines et des lipides diminue dès l'incorporation de $20 \%$ de lupin cru dans le régime. Toutefois, l'extrusion du lupin entraîne une augmentation de l'utilisation digestive de tous les nutriments, principalement de l'extractif non azoté ;

- après 83 jours d'alimentation, il y a une baisse des performances zootechniques chez la truite nourrie avec un régime contenant du LC ; mais chez les animaux qui reçoivent du LE1 ou LE2, la croissance, la consommation de nourriture, l'efficacité alimentaire et l'efficacité de rétention protéique sont nettement améliorées, ce qui laisse supposer une élimination, par l'extrusion, de facteurs antinutritionnels présents chez le lupin.
\end{abstract}

truite / lupin / extrusion / digestibilité / croissance

Summary - Nutritive value of white lupin (Lupinus albus var Lutop) fed to rainbow trout (Oncorhynchus mykiss). Effects of extrusion cooking. Two experiments were conducted in rainbow trout (Oncorhynchus mykiss) to assess the nutritional value of diets containing crude (LC) or extruded at $120^{\circ} \mathrm{C}$ (LE1) and $145^{\circ} \mathrm{C}$ (LE2) lupin var "Lutop" as a partial protein source. In the first experiment, the apparent digestibility coefficient (ADC) of diets with 20 and $30 \%$ lupin was measured. In a second trial, the effects of diet containing $20 \%$ crude and extruded lupin on growth performance of trout were analyzed. It was found that the $A D C$ of dry matter, proteins and lipids decreased with the incorporation of $20 \%$ crude lupin. The extrusion-cooking process increased the digestive utilization of nutrients, especially the nitrogen-free extract. After $83 d$ of feeding, decreased growth perfor-

\footnotetext{
* Correspondance et tirés à part
} 
mance was observed in trout fed on LC diet. Those fed diets LE1 or LE2 showed increased growth, food intake, food efficiency and protein retention, attributed to the disappearance of antinutritional factors in extruded lupin.

Oncorhynchus mykiss $=$ rainbow trout $/$ Lupinus albus = white /upin / extrusion / digestibility $/$ growth

\section{INTRODUCTION}

Dans un contexte mondial de demandes croissantes en matières premières riches en protéines, depuis plusieurs années, un grand effort de recherches est concentré sur la sélection et l'utilisation de protéagineux.

Parmi les protéagineux, le lupin est le mieux pourvu en protéines (30 à $40 \%$ de la matière sèche des graines) et si, pendant longtemps, son emploi en alimentation animale a été limité en raison de sa teneur en alcaloïdes, la sélection de lignées douces a entraîné de nombreuses expérimentations chez les animaux (Lacassagne, 1984 ; Hill, 1986).

Chez les poissons, les travaux réalisés chez la truite arc-en-ciel ont indiqué de bonnes performances de croissance et de conversion de l'aliment lorsque le taux d'incorporation de lupin doux dans le régime était de $26 \%$ dans le cas du lupin bleu (Gomes et Kaushik, 1989) et de 30 à $40 \%$ dans le cas du lupin blanc (De La Higuera et al, 1988 ; Hughes, 1988, 1991). Cependant, pour le lupin blanc doux, chez certains animaux (poulets, porcs) des résultats très divers ont été obtenus suivant la variété utilisée ; ces résultats étant partiellement expliqués par la teneur en alcaloïdes (Lacassagne, 1984 ; Hill, 1986). De La Higuera et al (1988) signalent qu'ils ont testé chez la truite la variété Multolupa, mais Hughes $(1988,1991)$ ne précise pas celle qu'il a employée, ce qui restreint, en conséquence, les données sur l'utilisation du lupin blanc doux dans l'alimentation de ce Salmonidé.
Bien qu'on ne connaisse pas de facteurs antinutritionnels thermolabiles chez le lupin, certaines études ont été consacrées à l'effet d'un traitement thermique (cuisson à la vapeur, passage à l'autoclave, cuisson-extrusion) sur sa valeur alimentaire chez des monogastriques (Mendes Pereira et Pion, 1984 ; Batterham et al, 1986a, 1986b ; Hill, 1986 ; De La Higuera et al, 1988). Seuls les essais réalisés avec du lupin blanc doux extrudé, effectués chez le porc et le rat, ont démontré une amélioration des performances zootechniques (Hill, 1986).

Le but de ce travail est de déterminer, pour la truite arc-en-ciel, la valeur nutritive du lupin blanc doux variété Lutop (sélectionné en France pour sa teneur élevée en protéines) et également de vérifier l'action de la cuisson-extrusion sur l'augmentation de l'efficacité alimentaire de cette Légumineuse.

\section{MATÉRIELS ET MÉTHODES}

\section{Traitement et composition chimique des farines de lupin}

Les graines de lupin blanc ont été fournies par la coopérative CCLP (Coopérative Couhé Luzignan Poitiers, 86700 Couhé). Elles ont été broyées en une farine dont une partie était conservée crue (LC), l'autre partie était extrudée (extrusion à sec - malaxeur-cuiseur-extrudeur type continua C37 - Werner et Pfleiderer) soit à $120^{\circ} \mathrm{C}$ à la pression de 12 bars pendant $35 \mathrm{~s}$ (LE1), soit à $145^{\circ} \mathrm{C}$ à la pression de 5 bars pendant $25 \mathrm{~s}$ (LE2). 
Par rapport à la matière sèche (MS), ces trois farines contiennent $38,50 \pm 1,02 \%$ de protéines brutes, $9,25 \pm 0,08 \%$ d'extraits d'éther, $4,00 \pm 0,07 \%$ de matières minérales et $20,87 \pm$ $0,94 \%$ de fibres brutes (dont $60 \%$ correspondent à de la cellulose). Leur composition en acides aminés essentiels (AAE) est similaire mais, comparée à celle de la farine de poisson, elle montre, excepté pour l'arginine, une déficience en ces composés, plus particulièrement en acides aminés soufrés (tableau I).

\section{Régimes expérimentaux}

Deux expériences furent réalisées : la première pour déterminer le coefficient d'utilisation digestive apparent (CUDa) du lupin (méthode indirecte avec $1 \%$ de $\mathrm{Cr}_{2} \mathrm{O}_{3}$ dans les aliments), et la seconde pour apprécier la croissance et les modifications de la composition corporelle des truites.

Les constituants et la composition chimique des régimes utilisés pour chaque expérience sont détaillés dans le tableau II.

Les aliments expérimentaux ont été formulés par remplacement du régime témoin par les fa- rines LC, LE1, LE2 au taux de 20 et $30 \%$ dans la première expérience, et uniquement au taux de $20 \%$ dans la seconde.

\section{Matériel animal}

Les truites utilisées provenaient d'une pisciculture régionale (pisciculture Les Viviers du Comminges Antignac - 31120 Bagnères de Luchon). Leur poids moyen initial était de $75 \mathrm{~g}$ pour la première expérience et de $50 \mathrm{~g}$ pour la seconde.

\section{Conditions expérimentales}

\section{Expérience I}

Cinq lots de 16 truites ont été placés dans des bacs cylindriques d'une capacité de 60 litres (dispositif de récolte des fécès décrit par Choubert et al, 1979), alimentés en eau du robinet $\left(11 \pm 2^{\circ} \mathrm{C}\right)$, à raison de $2 \mathrm{l} / \mathrm{min}$.

Pendant la période d'adaptation aux conditions de stabulation $(2 \mathrm{sem})$, les lots de pois-

Tableau I. Teneur en acides aminés essentiels (AAE) de la farine de poisson et des farines de lupin.

\begin{tabular}{|c|c|c|c|c|}
\hline \multirow{2}{*}{$\begin{array}{l}A A E \\
(g / 16 g N)\end{array}$} & \multirow{2}{*}{$\begin{array}{l}\text { Farine de } \\
\text { poisson * }\end{array}$} & \multicolumn{3}{|c|}{ Farines de lupin } \\
\hline & & LC & LE1 & LE2 \\
\hline Thréonine & 5,32 & 3,80 & 3,95 & 3,92 \\
\hline Valine & 5,65 & 3,80 & 3,97 & 3,83 \\
\hline Méthionine & 4,03 & 0,66 & 0,60 & 0,63 \\
\hline (Méthionine + cystine) & 4,52 & 1,99 & 1,90 & 1,85 \\
\hline Isoleucine & 4,69 & 3,86 & 4,10 & 3,90 \\
\hline Leucine & 8,73 & 6,56 & 6,85 & 6,58 \\
\hline Phénylalanine & 4,55 & 3,60 & 3,77 & 3,59 \\
\hline (Phenylalanine + tyrosine) & 8,21 & 7,31 & 7,59 & 7,26 \\
\hline Lysine & 8,20 & 5,49 & 5,74 & 5,45 \\
\hline Histidine & 2,21 & 2,02 & 2,06 & 2,05 \\
\hline Arginine & 6,50 & 9,80 & 10,1 & 9,89 \\
\hline
\end{tabular}

* Composition chimique (\%MS) : $69,50 \%$ de protéines brutes, $10,42 \%$ d'extraits d'éther et $16 \%$ de matières minérales. 
Tableau II. Constituants et composition chimique des aliments (\% MS).

\begin{tabular}{|c|c|c|c|c|c|}
\hline & \multicolumn{3}{|c|}{ Expérience I } & \multicolumn{2}{|c|}{ Expérience $/ 1$} \\
\hline & Témoin & $\begin{array}{c}20 \% \\
\text { (LC-LE1-LE2) }\end{array}$ & $\begin{array}{c}30 \% \\
(L C-L E 1-L E 2)\end{array}$ & Témoin & $\begin{array}{c}20 \% \\
\text { (LC-LE1-LE2) }\end{array}$ \\
\hline \multicolumn{6}{|l|}{ Constituants } \\
\hline Farine de poisson & 61,0 & 48,8 & 42,7 & 52 & 43,6 \\
\hline Farine de lupin & 0 & 20,0 & 30,0 & 0 & 20,0 \\
\hline Cellulose & 3 & 2,4 & 2,1 & 0 & 0 \\
\hline Amidon de maïs gélatinisé & 25 & 19,2 & 16,4 & 0 & 0 \\
\hline Remoulage blanc de blé* & 0 & 0 & 0 & 34 & 22,1 \\
\hline Huile de foie de morue & 6 & 4,8 & 4,2 & 10 & 9,5 \\
\hline Mélange vitaminique ${ }^{* *}$ & 2 & 1,6 & 1,4 & 2 & 2 \\
\hline Mélange minéra| $\left.\right|^{\star \star \star}$ & 2 & 1,6 & 1,4 & 2 & 2 \\
\hline DL-Méthionine & 0 & 0,6 & 0,8 & 0 & 0,8 \\
\hline $\mathrm{Cr}_{2} \mathrm{O}_{3}$ & 1 & 1 & 1 & 0 & 0 \\
\hline \multicolumn{6}{|l|}{ Composition chimique } \\
\hline $\begin{array}{l}\text { Protéines brutes } \\
(N \times 6,25)\end{array}$ & 42,3 & 41,6 & 41,7 & 42,0 & 42,0 \\
\hline Extraits d'éther & 12,1 & 11,3 & 11,3 & 17,0 & 16,8 \\
\hline Matières minérales & 12,9 & 11,6 & 10,6 & 11,7 & 10,6 \\
\hline
\end{tabular}

"Pour la préparation des aliments, le remoulage a été mélangé à l'eau, puis chauffé à $100^{\circ} \mathrm{C}$. ** $\mathrm{g} / 100 \mathrm{~g}$ de mélange: $\mathrm{B} 1: 0,3 ; \mathrm{B} 2: 0,5 ; \mathrm{B} 6: 0,3 ; \mathrm{B} 12: 0,0005 ; \mathrm{PP}: 2,0 ;$ acide folique $: 0,1 ; \mathrm{C}: 4,0 ;$ inositol $: 5,0:$ choline chloride $: 1,5$; biotine $: 0,0075 ; E: 1,0 ; \mathrm{K}: 0,3$; butyl-hydroxy-toluène $: 0,3 ;$ glucose $: 83,19 .{ }^{* *} \mathrm{~g} / 100 \mathrm{~g}$ de mélange : $\mathrm{NaCl}: 15 ; \mathrm{CaHPO}_{4}: 20 ; \mathrm{KHPO}_{4}: 30 ; \mathrm{CaCl}: 6 ; \mathrm{Mg} \mathrm{SO}_{4} ; 7 \mathrm{H}_{2} \mathrm{O}: 20 ; \mathrm{MnSO}_{4}, \mathrm{H}_{2} \mathrm{O}: 0,8 ; 4 \mathrm{MgCO}_{3}, \mathrm{Mg}(\mathrm{OH})$ $2,5 \mathrm{H}_{2} \mathrm{O}: 4,65 ; \mathrm{FeSO}_{4}: 1,75 ; \mathrm{ZnSO}_{4}, 7 \mathrm{H} 2 \mathrm{O}: 1,55 ; \mathrm{CuSO}_{4}, 5 \mathrm{H}_{2} \mathrm{O}: 0,25 ; \mathrm{IK}: 0,0085$.

sons ont été nourris avec de l'aliment commercial (AQUALIM, Groupe des grands moulins de Paris, 16440 Roullet-Saint-Estèphe $(48,06 \%$ de protéines brutes ; $9,31 \%$ de matières grasses et $12,14 \%$ de matières minérales) ( $2 \%$ du poids vif par j), puis ils ont reçu les différents régimes successivement $(2 \mathrm{j}$ de jeûne avant chaque changement de nourriture). Tous les régimes ont été distribués à satiété, deux fois par j $(9 \mathrm{~h}$ et $16 \mathrm{~h}$ ) durant $2 \mathrm{sem}$. Les fécès ont été récoltées quotidiennement à partir du $4^{\mathrm{e}} \mathrm{j}$ d'alimentation (12 j de collecte) ; elles ont été mises à sécher à l'étuve à vide sur chlorure de calcium et à la température ambiante, jusqu'à poids constant $(48 \mathrm{~h})$. Pour un même régime, les fécès d'un lot de truite ont été regroupées, broyées et gardées à $+4^{\circ} \mathrm{C}$ jusqu'aux analyses (4 analyses par lot).

\section{Expérience II}

Seize lots de 26 truites (4 répétitions $\times 4$ aliments) ont été placés dans des bassins de 2201 alimentés, en circuit ouvert, par de l'eau du robinet maintenue à $15 \pm 1^{\circ} \mathrm{C}$ et dont l'oxygénation était proche de la saturation.

Après la période d'adaptation (mêmes conditions que pour l'expérience 1), les poissons ont été mis à jeun durant $48 \mathrm{~h}$, puis pesés (temps initial). Les modalités d'alimentation des animaux étaient identiques à celles de la première expérience. Le poids des truites était évalué tous les $20 \mathrm{j}$. La durée de l'expérimentation était de $83 \mathrm{j}$.

Pour déterminer les modifications de la composition corporelle, des poissons ont été sacri- 
fiés au temps initial ( 1 individu par lot), et au temps final ( 3 individus par lot) de l'expérience, broyés individuellement, congelés dans de l'azote liquide et conservés à $-18^{\circ} \mathrm{C}$ jusqu'aux analyses.

\section{Méthodes analytiques}

La teneur en eau des matières premières et des aliments a été déterminée après passage à l'étuve à $130^{\circ} \mathrm{C}$ pendant $2 \mathrm{~h}$, et celle des broyats de poissons après déshydratation sous vide, sur chlorure de calcium à température ambiante. Les protéines brutes ont été évaluées par la méthode de Kjeldhal. Les lipides totaux des matières premières et des aliments ont été extraits par l'éther de pétrole à l'appareil de Soxhlet; pour les poissons, ils ont été dosés par la méthode de Folch et al (1957). Le taux des cendres a été calculé après calcination complète de l'échantillon sec à $550^{\circ} \mathrm{C}$ pendant $12 \mathrm{~h}$. Les fibres brutes du lupin ont été estimées par la méthode de Van Soest (1963) et Van Soest et Wine (1967). Après hydrolyse acide $(\mathrm{HCl} 6 \mathrm{M})$ des farines de lupin et de poisson, sous bullage d'azote à $137^{\circ} \mathrm{C}$ pendant $18 \mathrm{~h}$, leur composition en acides aminés a été déterminée par chromatographie sur résine échangeuse d'ions (Amino acid analyser $111 \mathrm{CL}$ Beckman). La teneur en $\mathrm{Cr}_{2} \mathrm{O}_{3}$ des fécès et des aliments a été dosée par la méthode de Bolin et al (1952).

La digestibilité de la matière sèche, des protéines $(P)$, des lipides $(L)$ et des cendres $(C)$ des farines de lupin (LC, LE1 et LE2) a été estimée à partir de la valeur des CUDa trouvée pour les aliments contenant $30 \%$ de farine, en utilisant la formule proposée par Cho et Slinger (1979) et Cho et al (1985) :

\section{(CUDa aliment expérimental $-0,7$ CUDa aliment témoin) / 0,3}

À partir des résultats obtenus et en considérant que la digestibilité des fibres (F) est négligeable (Hilton et al, 1983, Lésel et al, 1986), le CUDa de l'extractif non azoté (ENA) a été évalué par différence :

MSdigestible $-(P+L+C)$ digestibles

$100-(\% P+\% L+\% C+\% F)$

\section{Traitement statistique}

Les résultats ont été traités par analyse de variance et, le cas échéant, les différences significatives entre les moyennes ont été déterminées au seuil de $5 \%$ à l'aide du test de Tukey (Zar, 1984).

\section{RÉSULTATS}

\section{Expérience I}

Dès l'incorporation de $20 \%$ de LC dans l'aliment, on observe une diminution du CUDa de la matière sèche $(-5,5 \%)$ et, à un moindre dégré, de la digestibilité des protéines et des lipides (respectivement 0,9 et $-0,6 \%$ ) (tableau III). Une amélioration du CUDa des nutriments est notée après cuisson-extrusion du lupin, en particulier à $145^{\circ} \mathrm{C}$; en fait, il n'y a pas de différence significative entre les valeurs obtenues pour la digestibilité des protéines et des lipides de l'aliment LE2-20 et de l'aliment témoin, ce qui a conduit à retenir le taux de $20 \%$ de lupin dans les régimes pour la seconde expérience.

Le calcul des CUDa des farines de lupin (tableau IV) montre qu'après extrusion l'augmentation de l'utilisation digestive de I'ENA est nettement plus marquée que celle des protéines, lipides ou cendres.

\section{Expérience /I}

Après $41 \mathrm{j}$ d'alimentation avec un régime renfermant $20 \%$ de LC ou LE1 ou LE2, la croissance des truites est identique à celle du lot témoin, mais leur consommation alimentaire est légèrement plus élevée, d'où une hausse de l'indice de consommation (IC) et une baisse du coefficient d'efficacité protéique (CEP) (tableau V). Cependant, 
Tableau III. Coefficient d'utilisation digestive apparent (\%) des aliments renfermant 20 et $30 \%$ de LC ou LE1 ou LE2.

\begin{tabular}{|c|c|c|c|c|c|c|c|}
\hline & Témoin & $L C-20$ & $L C-30$ & $L E 1-20$ & $L E 1-30$ & LE2-20 & LE2-30 \\
\hline $\begin{array}{l}\text { Matière } \\
\text { sèche }\end{array}$ & $\begin{array}{l}71,5^{a} \\
\pm 0,5\end{array}$ & $\begin{array}{l}67,6^{c} \\
\pm 0,4\end{array}$ & $\begin{array}{l}65,6^{\mathrm{e}} \\
\pm 0,3\end{array}$ & $\begin{array}{l}69,5^{b} \\
\pm 0,2\end{array}$ & $\begin{array}{l}66,5^{d} \\
\pm 0,9\end{array}$ & $\begin{array}{l}69,8^{b} \\
\pm 0,2\end{array}$ & $\begin{array}{l}67,4^{c} \\
\pm 0,5\end{array}$ \\
\hline Protéines & $\begin{array}{l}90,0 \mathrm{a}^{2} \\
\pm 0,3\end{array}$ & $\begin{array}{l}89,2 b c \\
\pm 0,1\end{array}$ & $\begin{array}{l}88,9^{c} \\
\pm 0,2\end{array}$ & $\begin{array}{l}89,5^{b} \\
\pm 0,2\end{array}$ & $\begin{array}{l}89,0^{\mathrm{c}} \\
\pm 0,1\end{array}$ & $\begin{array}{l}89,6 \text { ab } \\
\pm 0,2\end{array}$ & $\begin{array}{l}89,4^{b} \\
\pm 0,2\end{array}$ \\
\hline $\begin{array}{l}\text { Extraits } \\
\text { d'éther }\end{array}$ & $\begin{array}{l}97,7 \mathrm{a} \\
\pm 0,4\end{array}$ & $\begin{array}{l}97,0^{\circ} \\
\pm 0,3\end{array}$ & $\begin{array}{l}96,6^{c} \\
\pm 0,2\end{array}$ & $\begin{array}{l}97,0^{b} \\
\pm 0,3\end{array}$ & $\begin{array}{l}96,9 \text { bc } \\
\pm 0,3\end{array}$ & $\begin{array}{l}97,4^{a} \\
\pm 0,2\end{array}$ & $\begin{array}{l}97,4^{a} \\
\pm 0,3\end{array}$ \\
\hline $\begin{array}{l}\text { Matières } \\
\text { minérales }\end{array}$ & $\begin{array}{l}38,2^{c} \\
\pm 0,5\end{array}$ & $\begin{array}{l}40,5^{b} \\
\pm 0,1\end{array}$ & $\begin{array}{l}40,5 b c \\
\pm 0,7\end{array}$ & $\begin{array}{l}40,9 b \\
\pm 0,1\end{array}$ & $\begin{array}{l}40,6^{b} \\
\pm 0,8\end{array}$ & $\begin{array}{l}41,5^{a} \\
\pm 0,3\end{array}$ & $\begin{array}{l}41,2 \text { ab } \\
\pm 0,5\end{array}$ \\
\hline
\end{tabular}

Valeur moyenne $\pm \mathrm{sd} ;{ }^{a}, b, c, d . e$ : différence significative entre les aliments $(P<0,05)$.

au terme de l'expérience $(83 \mathrm{j})$, si les résultats obtenus pour les animaux nourris avec un aliment contenant LE1 ou LE2 sont peu différents de ceux observés à la mi-période d'étude, une diminution de la croissance et de la prise de nourriture est remarquée chez les truites qui reçoivent le régime avec du LC. C'est d'ailleurs pour ces poissons que l'on note une importante mortalité dans les dernières semaines d'expérimentation (tableau V).

En fin d'expérience, aucune différence n'est décélée entre les lots de truites, dans

Tableau IV. Coefficient d'utilisation digestive apparent (\%) des farines de lupin.

\begin{tabular}{llll}
\hline & LC & LE1 & LE2 \\
\hline Matière sèche & 51,8 & 54,8 & 57,8 \\
Protéines & 86,3 & 86,7 & 88,0 \\
Extraits d'éther & 94,0 & 95,0 & 96,7 \\
Matières minérales & 45,9 & 46,2 & 48,2 \\
Extractif non azoté & 30,5 & 38,9 & 47,0 \\
\hline
\end{tabular}

la composition corporelle (tableau VI). Mais la valeur de l'efficacité de rétention protéique (ERP) décroît lorsque le lupin est incorporé dans l'aliment (par rapport au témoin : $-26 \%$ avec LC, $-15 \%$ avec LE1, $-13 \%$ avec LE2).

\section{DISCUSSION}

Chez la truite arc-en-ciel, la digestibilité apparente de l'aliment s'abaisse dès l'incorporation de $20 \%$ de lupin blanc "Lutop". Ce résultat est à rapprocher de celui de Gomes et Kaushik (1989) qui montre une diminution du CUDa de la matière sèche, des protéines et de l'énergie, lorsque le régime renferme plus de $13 \%$ de lupin bleu. Cependant, d'après De la Higuera et al (1988), il n'y pas d'altération de la digestibilité des protéines pour un taux d'incorporation de lupin blanc "Multolupa» de 40\%. On doit noter toutefois que ces auteurs ont obtenu les fécès par pression abdominale, alors que nous avons employé la même méthode de récolte que Gomes et Kaushik (1989). 
Tableau V. Croissance relative (CR), indice de consommation (IC), coefficient d'efficacité protéique (CEP) et mortalité chez la truite après 41 et 83 jours d'alimentation avec un régime renfermant $20 \%$ de LC ou LE1 ou LE2.

\begin{tabular}{|c|c|c|c|c|c|}
\hline & & Témoin & $L C$ & LE1 & LE2 \\
\hline $\begin{array}{l}\text { Poids moyen } \\
\text { initial }(g)\end{array}$ & & $\begin{array}{r}52,6 \\
\pm 7,2\end{array}$ & $\begin{array}{r}51,6 \\
\pm 8,9\end{array}$ & $\begin{array}{r}50,4 \\
\pm 7,7\end{array}$ & $\begin{array}{r}51,5 \\
\pm 8,0\end{array}$ \\
\hline Poids moyen $(\mathrm{g})$ & $\begin{array}{l}41 j \\
83 j\end{array}$ & $\begin{array}{r}118,5 \\
\pm 20,7 \\
179,6 \\
\pm 38,2\end{array}$ & $\begin{array}{c}110,9 \\
\pm 26,4 \\
146,6 \\
\pm 47,5\end{array}$ & $\begin{array}{r}116,4 \\
\pm 21,8 \\
176,1 \\
\pm 42,2\end{array}$ & $\begin{array}{r}114,6 \\
\pm 24,8 \\
180,4 \\
\pm 41,0\end{array}$ \\
\hline CR (\%) & $\begin{array}{l}41 j \\
83 j\end{array}$ & $\begin{array}{c}125,5^{a b} \\
\pm 8,9 \\
241,7 a \\
\pm 14,1\end{array}$ & $\begin{array}{c}114,8^{b} \\
\pm 6,7 \\
183,7^{b} \\
\pm 17,6\end{array}$ & $\begin{array}{l}131,2^{a} \\
\pm 4,4 \\
249,7^{b} \\
\pm 17,9\end{array}$ & $\begin{array}{c}122,7 \mathrm{ab} \\
\pm 8,3 \\
250,5 \mathrm{a} \\
\pm 15,7\end{array}$ \\
\hline $\begin{array}{l}\text { Consommation } \\
\text { (g/poisson) }\end{array}$ & $\begin{array}{l}41 j \\
83 j\end{array}$ & $\begin{array}{l}58,2^{b} \\
\pm 4,9 \\
123,0^{b} \\
\pm 5,6\end{array}$ & $\begin{array}{c}63,2^{a} \\
\pm 3,5 \\
110,8 \mathrm{c} \\
\pm 7,1\end{array}$ & $\begin{array}{c}65,8^{a} \\
\pm 1,2 \\
137,2^{a} \\
\pm 5,9\end{array}$ & $\begin{array}{c}63,2 \mathrm{a} \\
+1,9 \\
138,9 \mathrm{a} \\
\pm 6,9\end{array}$ \\
\hline IC & $\begin{array}{l}41 j \\
83 j\end{array}$ & $\begin{array}{c}0,88^{b} \\
\pm 0,04 \\
0,96 \mathrm{~b} \\
\pm 0,01\end{array}$ & $\begin{array}{c}1,06 \mathrm{a}^{\mathrm{a}} \\
\pm 0,08 \\
1,17 \text { a } \\
\pm 0,12\end{array}$ & $\begin{array}{c}0,99 \mathrm{a} \\
\pm 1,20 \\
1,09 \mathrm{~b} \\
\pm 0,04\end{array}$ & $\begin{array}{c}1,00^{a} \\
\pm 0,03 \\
1,08 \mathrm{~b} \\
\pm 0,01\end{array}$ \\
\hline CEP & $\begin{array}{l}41 j \\
83 j\end{array}$ & $\begin{array}{c}2,69 \mathrm{a} \\
\pm 0,13 \\
2,48 \mathrm{a} \\
\pm 0,05\end{array}$ & $\begin{array}{c}2,23^{\mathrm{b}} \\
\pm 0,15^{\circ} \\
2,04^{\mathrm{c}} \\
\pm 0,22\end{array}$ & $\begin{array}{c}2,39 a b \\
\pm 0,13 \\
2,19 b \\
\pm 0,09\end{array}$ & $\begin{aligned} & 2,38 \mathrm{ab} \\
& \pm 0,05 \\
& 2,23 \mathrm{~b} \\
& \pm 0,03\end{aligned}$ \\
\hline Mortalité (\%) & $\begin{array}{l}41 j \\
83 j\end{array}$ & $\begin{array}{l}2 \\
4\end{array}$ & $\begin{array}{r}8 \\
30\end{array}$ & $\begin{array}{l}2 \\
3\end{array}$ & $\begin{array}{l}2 \\
4\end{array}$ \\
\hline
\end{tabular}

Valeur moyenne $\pm \mathrm{sd} ; \mathrm{a}^{\mathrm{b}} \mathrm{b}, \mathrm{c}$ : différence significative entre les lots $(P<0,05)$.

Hugues (1988) indique que, chez la truite, le CUDa des protéines du lupin blanc (estimé en utilisant une chambre à métabolisme) est de $85,2 \%$. La valeur que nous avons obtenue pour ces composés est légèrement plus élevée $(86,3 \%)$. Cette différence peut être due à la méthode retenue pour l'étude de la digestibilité (Smith et al 1980) mais également à l'addition de
DL-méthionine au régime (Lopez de Romana, 1984).

La cuisson-extrusion du lupin blanc, en particulier à $145^{\circ} \mathrm{C}$, entraîne une augmentation de l'utilisation digestive des nutriments de ce protéagineux, principalement de l'ENA. Cet effet sur l'ENA pourrait être induit par une dégradation partielle des $\alpha$ galactosides présents dans la graine $(15 \%$ 
Tableau VI. Composition corporelle (\% poids frais) et efficacité de rétention protéique (ERP) chez la truite nourrie avec un aliment renfermant $20 \%$ de LC ou LE1 ou LE2.

\begin{tabular}{|c|c|c|c|c|c|}
\hline & \multirow[t]{2}{*}{ Temps initial } & \multicolumn{4}{|c|}{ Temps final } \\
\hline & & Témoin & $L C$ & $L E 1$ & LE2 \\
\hline Eau & $\begin{array}{r}76,9 \\
\pm 1,6\end{array}$ & $\begin{array}{r}65,9 \\
\pm 1,3\end{array}$ & $\begin{array}{r}67,0 \\
\pm 0,9\end{array}$ & $\begin{array}{r}66,2 \\
\pm 1,2\end{array}$ & $\begin{array}{r}66,6 \\
\pm 1,2\end{array}$ \\
\hline $\begin{array}{l}\text { Protéines brutes } \\
(\mathrm{N} \times 6,25)\end{array}$ & $\begin{array}{r}15,6 \\
\pm 0,8\end{array}$ & $\begin{array}{r}15,7 \\
\pm 0,6\end{array}$ & $\begin{array}{r}15,1 \\
\pm 0,6\end{array}$ & $\begin{array}{r}15,4 \\
\pm 0,4\end{array}$ & $\begin{array}{r}15,5 \\
\pm 0,6\end{array}$ \\
\hline Extraits d'éther & $\begin{array}{r}2,6 \\
\pm 0,8\end{array}$ & $\begin{array}{r}11,2 \\
\pm 1,2\end{array}$ & $\begin{array}{r}11,6 \\
\pm 0,6\end{array}$ & $\begin{array}{r}10,9 \\
\pm 0,9\end{array}$ & $\begin{array}{r}11,0 \\
\pm 0,8\end{array}$ \\
\hline Matières minérales & $\begin{array}{r}2,8 \\
\pm 0,2\end{array}$ & $\begin{array}{r}2,4 \\
\pm 0,1\end{array}$ & $\begin{array}{r}2,5 \\
\pm 0,2\end{array}$ & $\begin{array}{r}2,3 \\
\pm 0,1\end{array}$ & $\begin{array}{r}2,3 \\
\pm 0,2\end{array}$ \\
\hline \multicolumn{2}{|c|}{$\begin{array}{l}\text { ERP } \\
\text { (\% de protéines digérées) }\end{array}$} & $\begin{array}{l}43,5 \text { a } \\
\pm 0,9\end{array}$ & $\begin{array}{l}32,0^{\mathrm{c}} \\
\pm 2,0^{\circ}\end{array}$ & $\begin{array}{l}37,0^{b} \\
\pm 1,6\end{array}$ & $\begin{array}{l}38,0^{b} \\
\pm 0,6\end{array}$ \\
\hline
\end{tabular}

Valeur moyenne $\pm \mathrm{sd}$; a, b, c : différence significative entre les lots $(P<0,05)$.

de la matière sèche ; Saini, 1989). Une diminution de $30 \%$ de ces oligosaccharides a en effet été observée après extrusion, à température élevée, des graines de haricot et de lentilles (Melcion, 1987). De plus, pour tous les constituants du lupin, l'amélioration du CUDa peut résulter d'une plus grande accessibilité aux enzymes digestives grâce à la destruction des membranes de cellules par le traitement (Legoy, 1987).

L'étude des performances zootechniques des truites nourries avec le régime renfermant $20 \%$ de lupin révèle que, dans la seconde période d'expérience, la consommation alimentaire et la croissance des lots qui reçoivent du lupin cru sont plus faibles que celles des lots témoins.
De La Higuera et al (1988) avaient supposé que la présence, même à très faible taux, d'alcaloïdes et autres composés amers (saponines, flavonoïdes) dans les graines de "Multolupa" serait responsable de la diminution de la prise de nourriture observée, chez la truite alimentée durant 4 semaines, avec un régime contenant $40 \%$ de cette variété. Dans notre étude, l'appétence de l'aliment avec 20\% de "Lutop" non extrudé apparaît diminuer avec le temps. Toutefois, on peut également se demander si ces substances (teneur en alcaloïdes chez "Lutop" < $0,02 \%$ de MS aussi bien pour la lupanine que pour $\mathrm{I}^{\prime} \mathrm{OH}-$ lupanine et la spartéine; Razaka et al, 1992) ne sont pas responsables des résultats que nous avons obtenus, car l'inges- 
tion de nourriture des truites qui reçoivent un régime renfermant du lupin extrudé a toujours été supérieure à celle des lots témoins. Or, si un effet de la chaleur sur ces facteurs d'inappétence n'a jamais été décelé (Tomé et al, 1985; Van der Poel, 1989), d'après Legoy (1987) les composants amers de Légumineuses seraient éliminés à l'extrusion à sec au cours de la vaporisation flash d'eau qui se produit à la sortie de la filière de l'extrudeur.

L'augmentation de la prise alimentaire notée lorsque le lupin "Lutop" est incorporé aux régimes, soit durant les 41 premiers jours (LC), soit durant toute l'expérience (LE1 et LE2), est sans doute engendrée par la diminution des glucides digestibles dans l'aliment, entraînée par la réduction du pourcentage de remoulage de blé cuit. Chez la truite, un accroissement de la ration a été observé lorsque l'énergie digestible du régime est abaissée (Hilton et al, 1983 ; Bromley et Adkins, 1984 ; Kaushik et de Oliva Teles, 1985).

La plus faible valeur du CEP et de l'ERP obtenue lorsque l'aliment renferme du lupin pourrait d'ailleurs s'expliquer par une utilisation des protéines alimentaires à des fins énergétiques. Néanmoins, il est possible que la qualité des protéines soit également à l'origine de ce résultat. En effet, si dans nos essais la teneur en acides aminés essentiels des trois régimes expérimentaux est suffisante pour couvrir les besoins de la truite (Shanks et al, 1992 ; Kaushik, 1979 ; Ogino, 1980 ; Ketola, 1983), elle diffère quelque peu de celle de l'aliment témoin.

On constate cependant une diminution moins marquée du CEP et de l'ERP avec le lupin extrudé, ce qui pourrait être relié à la digestibilité de l'ENA; mais l'écart entre les quantités de glucides digestibles des aliments LE1, LE2 et LC est certainement minime. Cela conduit à supposer également l'existence de facteurs antinutrition- nels relativement thermolabiles dans les graines du lupin "Lutop».

La forte mortalité enregistrée chez les truites qui reçoivent un régime contenant du LC résulterait, ainsi, de l'effet dépressif de ces facteurs associé à une baisse de la consommation et de la digestibilité de l'aliment, et à une moindre qualité des protéines alimentaires. Cela demande toutefois à être vérifié.

\section{RÉFÉRENCES}

Batterham ES, Andersen LM, Lowe RF, Darnell RE (1986a) Effect of heat on the nutritional value of lupin (Lupinus angustifolius): seed meal for growing pigs. Br J Nutr 55, 169-177

Batterham ES, Andersen LM, Lowe RF, Darnell RE (1986b) Nutritional value of lupin (Lupinus albus) seed meal for growing pigs: availability of lysine, effect of autoclaving and net energy content. Br J Nutr 56, 645-659

Bolin W, King RP, Klosterman EW (1952) A simplified method for determination of chromic oxide $\left(\mathrm{Cr}_{2} \mathrm{O}_{3}\right)$ when used as an index substance. Science 116, 634-635

Bromley PJ, Adkins TC (1984) The influence of cellulose filler on feeding, growth and utilization of protein and energy in rainbow trout (Salmo gairdneri Rich). J Fish Biol 24, 235244

Choubert G, De La Noue J, Luquet P (1979) Continuous quantitative automatic collector for fish feces. Prog Fish Cult 1 (2), 64-67

Cho CY, Slinger SJ (1979) Apparent digestibility measurement in feedstuffs for rainbow trout. In: Finfish nutrition and fishfeed technology, vol I/ (JE Halver, K Tiews eds), Heenemann Gmbh et Co, Berlin, 239-249

Cho CY, Cowey CB, Watanabe T (1985) In: Finfish nutrition in asia: methodological approaches to research and development. Ottawa, Ont, IRDC, 154 p: ill

De La Higuera M, Garcia-Gallego M, Sanz A, Cardenete G, Suarez MD, Moyano FJ (1988) Evaluation of lupin seed meal as an alternative protein source in feeding rainbow trout (Salmo gairdneri). Aquaculture 71, 37-50

Folch J, Lees M, Stanley S (1957) A simple method for the isolation and purification of to- 
tal lipids from animal tissues. $\mathrm{J}$ Biol Chem 226, 497-509

Gomes EF, Kaushik SJ (1989) Incorporation of lupin seed meal, colzapro or tritical as protein/energy substitutes in rainbow trout diets. In: Proc 3rd int symp on feeding and nutr in fish. Toba, Aug 28-Sept 1, Japan, 315-324

Hill GD (1986) Recent developments in the use of lupin in animal and human nutrition. In: Proc 4th int Lupin Conf. Geraldton, 15-22 August, Western Australia, 40-63

Hilton SW, Atkinson JL, Slinger SJ (1983) Effect of increased dietary fiber on the growth of rainbow trout (Salmo gairdneri). Can J Fish Aquat Sci 40, 81-85

Hughes SG (1988) Assessment of lupin flour as a diet ingredient for rainbow trout (Salmo gairdneri) Aquaculture 43, 1149-1155

Hughes SG (1991) Use of lupin flour as a remplacement for full-fat soy in diets for rainbow trout (Oncorhynchus mykiss). Aquaculture 93, 57-62

Kaushik SJ (1979) Application of a biochemical method for the estimation of amino acid needs in fish: quantitative arginine requirements of rainbow trout in different salinities. In: Finfish nutrition and fishfeed technology, vol I (JE Halver, K Tiews ed) Heenemann Gmbh et Co, Berlin, 197-207

Kaushik SJ, de Oliva Teles A (1985) Effect of digestible energy on nitrogen and energy balance in rainbow trout. Aquaculture 50, 89-101

Ketola HG (1983) Requirement for dietary lysine and arginine by fry of rainbow trout. J Anim Sci 56, 101-107

Lacassagne $L$ (1984) Valeur nutritive du lupin blanc doux en alimentation animale. $/ n$. Actes du $3^{e}$ Congrès Int du Lupin. La Rochelle, juin 4-8, France, 421-451

Legoy $M$ (1987) L'extrusion à sec en alimentation animale conventionnelle : intérêt technique et économique, réalisation, perspective. In : Cuisson-extrusion, Nantes, 16-17 septembre 1986 ( $P$ Colonna ed) INRA, Paris, 267-275

Lésel R, Fromageot $C$, Lésel $M$ (1986) Cellulose digestibility in grass carp, Ctenopharyngodon idella and in goldfish, Carassius auratus. Aquaculture 54, 11-17

Lopez De Romana G (1984) Utilisation du lupin en alimentation humaine. $\mathrm{ln}$ : Actes $d u 3^{e}$ congrès int du lupin. La Rochelle, juin 4-8, France, 453-463

Melcion JP (1987) Oléo-protéagineux et cuisson-extrusion. In: Cuisson-extrusion, Nantes, 16-17 septembre 1986 (P Colonna, ed) INRA, Paris, 235-248

Mendes Pereira E, Pion R (1984) Digestive and metabolic utilization of lupin amino acid: effect of heat. In: Actes du $3^{e}$ Congr int du lupin, La Rochelle, juin 4-8, France, 645-646

Ogino C (1980) Requirements of carp and rainbow trout for essential amino acids. Bull Jpn Soc Sci Fish 46 (2), 171,-174

Razaka E, Pothiers J, Moncoulon R (1992) Effect of quinolizidinic alkaloids in lupin seeds: $L$ mutabilis and $L$ albus on organic and nitrogen ruminal degradation in dairy cows. In: Actes de la lre Conf Eur sur les protéagineux. Angers, 1-3 juin, 507-508

Saini HS (1989) Legume seed oligosaccharides. In: Recent advances of research in antinutritional factors in legume seeds (J Huismann, TFB van der Poel, IE Liener eds), Wageningen, The Netherlands, 329-341

Shanks WE, Gahimer GD, Halver JE (1962) The indispensable amino acids for rainbow trout. Prog Fish Cult 24, 68-73

Smith RR, Peterson MC, Allred AC (1980) Effect of leaching on apparent digestion coefficients of feedstuffs for Salmonids. Prog Fish Cult 42 (4), 195-199

Tome D, Valdebouze P, Krempf M (1985) Les principales actions des composés indésirables associés aux protéines végétales. $I n$ : Protéines végétales. Lavoisier ed, Paris, 309333

Van der Poel AFB(1989) Effects of processing on antinutritional factors (ANF) and nutritional value of legume seeds for non-ruminant feeding. In: Recent advances of research in antinutritional factors in legume seeds ( $\mathrm{J}$ Huissman, TFB Van der Poel, IE Liener eds) Wageningen, The Netherlands, 213-229

Van Soest PJ (1963) Use of detergent in the analysis of fibrous feeds. I. A rapid method for determination of fiber and lignin. J Assoc Off Agric Chem 46, 829-835

Van Soest PJ, Wine RH (1967) Use of detergent in the analysis of fibrous feeds. II. Determination of plants cell wall constituants. J Assoc Off Agric Chem 50, 50-55

Zar JH (1984) Biostatistical analysis. Prentice Hall Int, Londres, pp 718 